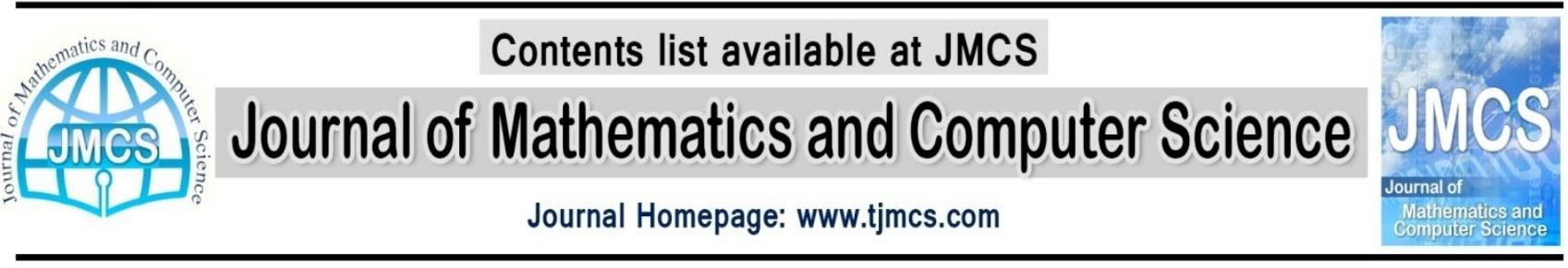

\title{
Some Notes on the Convergence Control \\ Parameter in the Framework of the Homotopy \\ Analysis Method
}

Jamshid Saeidian and Shahnam Javadi

Faculty of Mathematical Sciences and Computer, Kharazmi University, 50 Taleghani avenue, Tehran 1561836314, Iran.

j.saeidian@khu.ac.ir

Article history:

Received August 2013

Accepted November 2013

Available online November 2013

\section{Abstract}

The convergence control parameter and the technique of $\mathrm{c}_{0}$-curves is an unavoidable part of any homotopy analysis method work. The mathematical background of this parameter has been studied by other authors. Here we revisit this parameter and its essence; we mention that in some examples the parameter may fail to work. Also we give some comments in using the technique of $\mathrm{c}_{0}$-curves and show, through examples, that a misusage may lead the user to wrong results.

Keywords: Homotopy analysis method; Convergence-control parameter; Technique of $\mathrm{c}_{0}$-curves.

\section{Introduction}

Homotopy analysis method (HAM) is an efficient method for handling various kinds of functional equations like PDEs $[10,18,21]$, ODEs [22,23,5], BVPs [1], systems of equations [14,3], fractional forms $[17,12,20]$, integral equations [15] and many other families and it actually is a technique specialized for nonlinear equations $[21,23]$. Other than applied mathematicians, the method has found its way into different fields in engineering and sciences, for example chemists, physicians, biologists also take advantage of this powerful analytic technique for coping their own problems and equations. One can, also, find HAM footprints in other fields like finance problems [19].

As well as its applications, it is a method which is well-established from a mathematical point of view, Liao and his colleagues have successfully tried to enrich the method $[9,10]$. Others have tried to find its relation with classic methods and give a general algorithm for its implementation, the interested reader would find good references in $[7,8]$.

This amount of applications and literature and the global interest and effort among researchers is a witness of its great potential and unavoidable contribution to the community. 
In the framework of the HAM, if the equation under study has a solution, which has a series representation, then HAM is able to give a good approximation of this solution. HAM is equipped with a convergence control parameter (CCP) [7] and the final representation of the solution is dependent upon this parameter. Using this parameter one can easily control and possibly extend the convergence region of the solution obtained; this advantage makes HAM a favorite technique for applied mathematicians.

It has been proved that the HAM solution with this CCP is a Taylor series expansion of the exact solution at some point [11]. The Taylor series expansions usually have restricted convergence regions which makes them useless in some applications; the CCP is a gift of HAM to the community of analytic and semi-analytic methods which can easily overcome this lack.

Although CCP is generally accepted in the HAM community and it has enriched the HAM, there are still open questions about it that should be discussed:

- Is CCP able to control and extend the convergence region of HAM solution to any equation?

- Is the technique of $\mathrm{c}_{0}$-curves an efficient method to find suitable values of the CCP in any case?

These are sample and general questions which haven't been explicitly answered yet. In this work we would address similar questions and challenges concerning CCP and try to answer them to some extent.

The focus of this paper is on the CCP which is just one of the four well-known auxiliary elements in HAM. For discussions and similar works on other auxiliary elements, that HAM is equipped with, the interested reader can refer to $[13,15]$.

The early HAM which was proposed in [21], didn't have any CCP and this parameter is added to the homotopy equation in 1997 [4]. At the same time, Liao proposed a numerical method called "the technique of $\mathrm{C}_{0}$-curves" (TCC) to find acceptable and suitable values of the CCP, since then the parameter and the technique have extensively been used by HAM workers. The $\mathrm{c}_{0}$-curves and its results is an unavoidable section of any HAM work.

Despite general approval of the TCC, till 2011 there was neither an explicit mathematical reasoning, nor a detailed discussion for this powerful tool. In 2011 Abbasbandy et. al. [2] proved that the TCC does have a powerful mathematical background and once it is used carefully it would give us the desired convergence region. However there are still gaps that should be filled in. The main concerns of the authors in this work are:

- Is the CCP able to extend the convergence region of the solution series of any equation which is solved by HAM?

- How can one calculate the suitable values of the CCP?

- How much promising is the TCC?

The first two questions are discussed in section 2 and section 3 is devoted to the third question.

\section{Role of the CCP in HAM}

Expected from its name, the CCP is able to control and extend the convergence region of the series solution obtained by HAM, i.e. the HAM solution. Following examples clarify this ability. 
Example 2. 1. Consider the simple first order ordinary differential equation $u^{\prime}=u^{2}$ with the initial condition $u(0)=1$, which leads to the exact solution $u(x)=\frac{1}{1-x}$.

Setting the auxiliary operator to be $\mathcal{L}=\frac{d}{d x}$ and $u_{0}=0$ as the initial guess, we apply the HAM. If we accept that the solution series is absolutely convergent, rearranging the terms we have the following representation for the solution:

$$
\begin{aligned}
u(x)= & u_{0}(x)+u_{1}(x)+u_{2}(x)+u_{3}(x)+\cdots \\
& 1-c_{0} x+c_{0}^{2} x^{2}-c_{0}\left(1+c_{0}\right) x-c_{0}^{3} x^{3}+2 c_{0}^{2} x^{2}+2 c_{0}^{3} x^{2}-c_{0} x-2 c_{0}^{2} x-c_{0}^{3} x+\cdots \\
= & 1-c_{0} x\left(1+\left(1+c_{0}\right)+\left(1+c_{0}\right)^{2}+\left(1+c_{0}\right)^{3}+\cdots\right) \\
& +c_{0}^{2} x^{2}\left(1+2\left(1+c_{0}\right)+3\left(1+c_{0}\right)^{2}+4\left(1+c_{0}\right)^{3}+\cdots\right) \\
& -c_{0}^{3} x^{3}\left(1+3\left(1+c_{0}\right)+6\left(1+c_{0}\right)^{2}+12\left(1+c_{0}\right)^{3}+\cdots\right) \\
& \vdots \\
= & 1+x\left(\frac{-c_{0}}{1-\left(1+c_{0}\right)}\right)+x^{2}\left(\frac{c_{0}^{2}}{\left(1-\left(1+c_{0}\right)\right)^{2}}\right)+x^{3}\left(\frac{-c_{0}^{3}}{\left(1-\left(1+c_{0}\right)\right)^{3}}\right)+\cdots \\
= & 1+x+x^{2}+x^{3}+\cdots \\
= & \frac{1}{1-x},
\end{aligned}
$$

of course we accepted that $x \in(-1,1)$. We see that the suitable values of $c_{0}$ lie in the interval $(-2,0)$.

Example 2. 2. Consider the nonlinear diffusion equation $u_{t}=\left(u u_{x}\right)_{x}$, with the initial condition $u(x, 0)=x^{2}$. The solution is $u(x, t)=\frac{x^{2}}{1-6 t}$. Choosing the auxiliary linear operator $\mathcal{L}=\frac{\partial}{\partial t}$ and $u_{0}=0$, in the framework of HAM, the $m$ th order approximation reads

$$
\begin{aligned}
\operatorname{app}_{m}(x, t) & =u_{0}(x, t)+u_{1}(x, t)+\cdots+u_{m}(x, t) \\
& =x^{2}+\sum_{n=1}^{m}-6 c_{0} t x^{2}\left(-6 c_{0} t+\left(1+c_{0}\right)\right)^{n-1} .
\end{aligned}
$$

First we consider the $c_{0}=-1$ case, which is a special case known to be the homotopy perturbation method, 


$$
\begin{aligned}
& u_{0}(x, t)=x^{2}, \\
& u_{1}(x, t)=6 t x^{2}, \\
& u_{n}(x, t)=(6 t)^{n} x^{2}, \quad n \geq 2 .
\end{aligned}
$$

So we have

$$
\operatorname{app}_{m}(x, t)=u_{0}(x, t)+u_{1}(x, t)+\cdots+u_{m}(x, t)=x^{2}\left(1+6 t+\cdots+(6 t)^{m}\right),
$$

which results in

$$
\lim _{m \rightarrow \infty} \operatorname{app}_{m}(x, t)=x^{2}\left(1+6 t+(6 t)^{2}+\cdots\right) .
$$

As far as $t$ satisfies $\frac{-1}{6}<t<\frac{1}{6}$, this series converges to $u(x, t)=\frac{x^{2}}{1-6 t}$, which is the exact solution we look for. So in the special case $c_{0}=-1$, HAM gives a series which converges to the exact solution for all $x$ and $\frac{-1}{6}<t<\frac{1}{6}$. However this region is relatively a small one.

Now we turn to the general case, where we leave $c_{0}$ undetermined. The $m$-term approximation, i.e. $a p p_{m}$, is a geometric series which is convergent whenever $\left|1+c_{0}-6 c_{0} t\right|<1$. With this condition $\operatorname{app}_{m}$ converges to

which is the exact solution.

$$
x^{2}-6 c_{0} t x^{2} \frac{1}{1-\left(1+c_{0}-6 c_{0} t\right)}=\frac{x^{2}}{1-6 t},
$$

Two separate cases could be considered:

1- If $c_{0}>0$ then $\left|1+c_{0}-6 c_{0} t\right|<1$ results in $\frac{1}{6}<t<\frac{1}{6}+\frac{1}{3 c_{0}}$, so the convergence region of the solution series, in $c_{0}>0$ case, would be

$$
\left\{\begin{array}{c}
x \in \mathbb{R} \\
\frac{1}{6}<t<\frac{1}{6}+\frac{1}{3 c_{0}} .
\end{array}\right.
$$

Here it is possible to extend the convergence region by choosing smaller values of $c_{0}$. However positive $c_{0} \mathrm{~s}$ can not guarantee the convergence of the solution series for $t<\frac{1}{6}$, for these values of $t$ we refer
to negative $c_{0} \mathrm{~s}$.

2-If $c_{0}<0$ then the condition $\left|1+c_{0}-6 c_{0} t\right|<1$, would be equivalent to $\frac{1}{6}+\frac{1}{3 c_{0}}<t<\frac{1}{6}$, so it is possible to satisfy convergence by suitable negative $c_{0} \mathrm{~s}$.

One question arises here: Is the CCP can extend the convergence region of any HAM solution?

As best of our knowledge, there is no clear answer to this question. However we show that the answer would be "No", at least in the case of systems of linear equations. The upcoming example shows that when we are faced with linear equations, the CCP may not work. 
Example 2. 3. Consider the system of linear equations, $A x=b$, where $A$ is an invertible matrix of size $n$ and $x, b \in \mathbb{R}^{n}$. Suppose $v^{\left({ }^{\circ}\right)}$ is an initial guess of the solution, using an auxiliary matrix ${ }^{1} M$ (probably related to $A$ ), we construct a homotopy as follows:

$$
(1-q) M\left(x-v^{(\circ)}\right)+c_{0} q(A x-b)=0,
$$

where $c_{0}$ is the CCP.

According to HAM the resulted system reads:

$$
\begin{aligned}
& M\left(x^{\left({ }^{\circ}\right)}-v^{\left({ }^{\circ}\right)}\right)=0, \\
& M x^{(1)}=c_{0}\left(b-A x^{\left({ }^{\circ}\right)}\right), \\
& M x^{(n)}=\left(M-c_{0} A\right) x^{(n-1)}, \quad n \geq 2 .
\end{aligned}
$$

The convergence condition for this method would be $\rho\left(I-\alpha M^{-1} A\right)<1$.

So the main question we are concerned with, can be stated as follows:

"In solving a system of equations $A x=b$, using the homotopy method, can we ensure the convergence by suitably choosing the auxiliary matrix $M$ and the auxiliary parameter $c_{0}$ ?"

If the answer to this question is "Yes", then for an arbitrary matrix $B$ we must be able to find a scalar $c_{0}$ such that $\rho\left(I-c_{0} B\right)<1$.

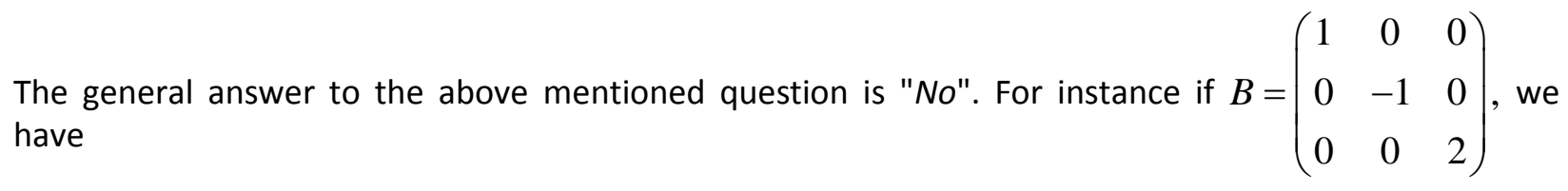

$$
I-c_{0} B=\left(\begin{array}{ccc}
1-c_{0} & 0 & 0 \\
0 & 1+c_{0} & 0 \\
0 & 0 & 1-2 c_{0}
\end{array}\right)
$$

So $\rho\left(I-c_{0} B\right)=\max \left\{\left|1-c_{0}\right|,\left|1+c_{0}\right|,\left|1-2 c_{0}\right|\right\}>1$.

\subsection{Evaluating the CCP}

The HAM output is a series with respect to a $c_{0}$. For certain values of $c_{0}$ the series would converge to the desired solution, we denote these values with $D_{c_{0}}$.

\footnotetext{
${ }^{1}$ same as "preconditioning matrix ", but here we preferred to use the term "auxiliary" as it is commonly used in a homotopy method framework.
} 
Definition 2.1. The values of the CCP for which the HAM series solution is convergent is called the convergence region of the CCP and it is denoted by $D_{c_{0}}$, i.e. whenever $c_{0} \in D_{c_{0}}$ we have a convergent series.

Now the main question can be rephrased as:" How one can determine $D_{c_{0}}$ ?"

In Example 2.1 we saw that $D_{c_{0}}=(-2,0)$, this means that for every $c_{0} \in(-2,0)$ the homotopy series would converge to the exact solution [22]. On the other hand, for the linear case in Example 2.3 we had $D_{c_{0}}=\varnothing$, which means that no value of the CCP results in a convergent series.

Unfortunately, in most of the examples it is not so easy to compute $D_{c_{0}}$ and in some cases it seems to be impossible to have an explicit formula for $D_{c_{0}}$, therefore a numerical approach is unavoidable.

So far the only numerical method is the well-known TCC. TCC is proposed in 1997, almost the same time when CCP was introduced to the homotopy equation and since then it has largely been used by HAM users. The approximation resulted from HAM is in fact a truncation of the generalized Taylor series of the exact solution [11] which depends upon a CCP, let's denote this approximation by $\operatorname{app}_{m}(\mathbf{r}, t)$, where $m$ is the order of approximation and $\mathbf{r}$ and $t$ denote spatial and time variables respectively.

Liao asserts that if HAM has done its work properly then the plot of $\operatorname{app}_{m}\left(\mathbf{r}_{\mathbf{0}}, t_{0}\right)$, for some $\mathbf{r}_{\mathbf{0}}$ and $t_{0}$ in the domain, as a function of $c_{0}$ must have a horizontal line. The values of $c_{0}$ corresponding to the horizontal line is denoted by $R_{c_{0}}$. According to Liao for any $c_{0} \in R_{c_{0}}$ the solution series obtained from HAM would converge to the exact solution of the equation under study.

Now the main question is that whether $R_{c_{0}}$ suitably approximates $D_{c_{0}}$ or not. This question is discussed in the next section. In 2011 Abbasbandy et al. [2] proved that the TCC is a promising method that can efficiently approximate $D_{c_{0}}$.

\subsection{Some Numerical notes}

In applying HAM and using the CCP there are some notes that should be considered by users, especially non-mathematicians. Adding $c_{0}$ to the homotopy equation increases the computational complexity of the problem and the process gets more complicated and time-consuming when $c_{0}$-curves should be plotted, so it would no more be a hand-pencil method. Actually HAM is a method of this age, the Computer Age, we have high-speed CPUs and powerful soft wares (like Mathematica, Maple and Matlab) that can do millions of operations in seconds, and as Liao states [21]: "The concept of analytic solution should be updated and in the time of computer, an analytic expression is not absolutely necessary to be only a few terms. In this meaning, the homotopy analysis method is for the time of computer, more or less."

However if the calculations are done with a wrong degree of precision then the results may not coincide reality. One can simply check this fact through any example. For instance in solving the ODE $y^{\prime}=2 y \quad(y(0)=1)$ via HAM in Maple15, when we use 10 digits of precision (which is Maple's default)

the 40th approximation doesn't have any horizontal line in its graph (we used a 4 digit decimal precision for $y$-axis). But when the precision is increased to 20 digits the resulted $R_{c_{0}}$ is close enough to $D_{c_{0}}$. This is because other than the precision degree of the software, one should also be careful in determining the horizontal line in a $c_{0}$-curve, i.e. $R_{c_{0}}$. For a suitable tolerance $\epsilon$, those $c_{0} \mathrm{~s}$ who satisfy $\left|\operatorname{app}_{m}\left(\mathbf{r}_{\mathbf{0}}, t_{0}\right)-u_{e}\left(\mathbf{r}_{\mathbf{0}}, t_{0}\right)\right|<\epsilon$ (where $u_{e}$ denotes the exact solution and of course not at hand!) are 
reported as $R_{c_{0}}$. A coarse choice of $\epsilon$ may result in an interval which is far from $D_{c_{0}}$, in other hand a very small $\epsilon$ may show no horizontal line in its graph. This is quite reasonable because $\operatorname{app}_{m}\left(\mathbf{r}_{\mathbf{0}}, t_{0}\right)$ is in fact a polynomial of $c_{0}$ and any polynomial of large degree may have an oscillatory graph.

\section{How much promising is the TCC?}

Although the theorem in [2] proves the mathematical reasoning of the TCC, there are still unknown and vague points in its implementation which should be carefully discussed. One should be very careful about numerical implementation and the notes which have been pointed out in section 2.2. To use TCC one has to decide on some elements in advance, which can be listed as:

- the order of approximation needed for plotting the $c_{0}$-curves,

- the quantity used to plot a $c_{0}$-curve,

- $\quad \mathbf{r}_{\mathbf{0}}$ and $t_{0}$, i.e. the points where $\operatorname{app}_{m}(\mathbf{r}, t)$ should be calculated to plot the $c_{0}$-curve.

No reference in HAM gives an explicit clue on how to choose these elements. Liao asserts that the type of the quantity is of less importance [10]. This is reasonable because once we accept that the HAM solution is a generalized Taylor series at some point [11] then any quantity concerning a derivative would simply relate to the original function through differentiation. In the case of uniform convergence the convergence of the series results in the convergence of its derivative, this fact makes no difference between quantities used to draw $c_{0}$-curves.

The order of approximation is the next case that should be considered, in fact any HAM user knows that adding the CCP to the homotopy equation extensively increases the computational cost, especially in PDEs and in plotting $c_{0}$-curves, so it makes no advantage if large values of $m$, order of approximation, satisfies the requirements of the theorem in [2].

What comes out from that theorem is that larger values of $m$ results in better approximations and $D_{c_{0}}$ is better approximated by $R_{c_{0}}$. This is true as long as one takes good care of the numerical points mentioned in section 2.2, otherwise the results may mislead the user.

The point $\left(\mathbf{r}_{0}, t_{0}\right)$ plays an important role and it should be chosen carefully. Having a knowledge about the domain of the equation and type of the solution (which is needed for Liao's rule of solution expression [22]) or any other physical background will help in this choice. Here we discuss this fact through Example 2.1. We used Maple 15 with 50 digits precision to obtain $a_{p p} p_{40}$ and used the approximation itself to plot $c_{0}$-curves at different points. For $x_{0}=0.1$ the TCC results in $R_{c_{0}}=(-1.95,0.25)$ which is reasonable. But for $x_{0}=0.5 \quad c_{0}$-curves reflect the interval $(-3.5,-0.5)$ which is wrong. Moreover if one uses $x_{0}=1$ the TCC fails to report any $R_{c_{0}}$ and for $x_{0}=2$ it would result in the wrong interval $(0.2,1.7)$.

It is really important to choose the above mentioned elements carefully and a wrong choice may mislead the user.

\section{AKNOWLEDGEMENT}

The authors would like to thank Miss B. Farahani for helping us in programming and checking the results. 


\section{References}

\subsection{Journal Article}

[1] S. Abbasbandy, E. Magyari, E. Shivanian, The homotopy analysis method for multiple solutions of nonlinear boundary value problems, Commun. Nonlinear Sci. Numer. Simul. 14 (2009) 3530-3536.

[2] S. Abbasbandy, E. Shivanian, K. Vajravelu, Mathematical properties of \$ \hbar\$-curve in the frame work of the homotopy analysis method, Commun. Nonlinear Sci. Numer. Simul. 16 (2011) 4268-4275.

[3] M. Fardi, K. Sayevand, Homotopy analysis method: a fresh view on Benjamin-Bona-Mahony-Burgers equation, Journal of Math. Computer Sci. 4 (2012) 494-501.

[4] S.J. Liao, A kind of approximate solution technique which does not depend upon small parameters (II): an application in fluid mechanics, Int. J. Non-Linear Mech. 32 (1997) 815-822.

[5] S.J. Liao, On the homotopy analysis method for nonlinear problems, Appl. Math. Comput. 147 (2004) $499-513$.

[6] S.J. Liao, Comparison between the homotopy analysis method and homotopy

perturbation method, Appl. Math. Comput. 169, 1186 (2005).

[7] S.J. Liao, Notes on the homotopy analysis method: Some definitions and theorems, Commun. Nonlinear Sci. Num. Simul. 14 (2009) 983-997.

[8] S.J. Liao, An optimal homotopy-analysis approach for strongly nonlinear differential equations. Commun. Nonlinear Sci. Num. Simul. 15 (2010) 2003-2016.

[9] S.J. Liao, A.T. Chwang, Application of homotopy analysis method in nonlinear oscillations,

ASME. J. Appl. Mech, 65 (1998) 914-922.

[10] S.J. Liao, Y. Tan, A general approach to obtain series solutions of nonlinear differential equations, Stud. Appl. Math. 119 (2007) 297-354.

[11] C.S. Liu, The essence of the generalized Taylor theorem as the foundation of the homotopy analysis method, Commun. Nonlinear Sci. Numer. Simul. 16 (2011) 1254-1262.

[12] Z. Odibat, S. Momani, H. Xu, A reliable algorithm of homotopy analysis method for solving nonlinear fractional differential equations, Appl. Math. Modell. 34 (2010) 593-600.

[13] S.J. Ramazannia Toloti , J. Saeidian, Comments on "R.A. Van Gorder and K. Vajravelu, Commun. Nonlinear Sci. Numer. Simul. 14 (2009) 4078-4089", Commun. Nonlinear Sci. Num. Simul. 17 (2012) 1085-1088.

[14] A. Sami Bataineh, M.S.M. Noorani, I. Hashim, Modified homotopy analysis method for solving systems of second-order BVPs, Commun. Nonlinear Sci. Num. Simul. 14 (2009) 430-442.

[15] R.A. Van Gorder, K. Vajravelu, On the selection of auxiliary functions, operators and convergence control parameters in the application of the Homotopy Analysis Method to nonlinear differential equations: A general approach, Commun. Nonlinear Sci. Numer. Simul. 14 (2009) 4078-4089.

[16] H. Vosughi, E. Shivanian, S. Abbasbandy, A new analytical technique to solve Volterra's integral equations, Math. Meth. Appl. Sci. 34 (2011) 1243-1253.

[17] H. Xu, S.J. Liao, X.C. You, Analysis of nonlinear fractional partial differential equations with the homotopy analysis method, Commun. Nonlinear Sci. Numer. Simul. 14 (2009) 1152-1156.

[18] A. Zare, M.A. Firoozjaee, Numerical solution for Maxwell's equation in metamaterials by homotopy analysis method, Journal of Math. Computer Sci. 3 (2011) 225-235.

[19] S.P. Zhu, An exact and explicit solution for the valuation of American put options, Quantitative Finance, 6 (2006) 229-242.

[20] M. Zurigat, S. Momani, Z. Odibat, A. Alawneh, The homotopy analysis method for handling systems of fractional differential equations, Appl. Math. Modell. 34 (2010) 24-35.

\subsection{Book}

[21] S.J. Liao, Proposed homotopy analysis techniques for the solution of nonlinear problems, Ph.D. dissertation, Shanghai Jiao Tong University, 1992.

[22] S.J. Liao, Beyond perturbation: An introduction to homotopy analysis method, Boca Raton: Chapman Hall/CRC Press; 2003.

[23] S.J. Liao, Homotopy analysis method in nonlinear differential equations, Springer and Higher Education Press, Heidelberg, 2012. 